\title{
Molecular Properties of Symmetrical Networks Using Topological Polynomials
}

https://doi.org/10.1515/chem-2019-0109

Received June 25, 2018; accepted March 21, 2019

\begin{abstract}
A numeric quantity that comprehend characteristics of molecular graph $\Gamma$ of chemical compound is known as topological index. This number is, in fact, invariant with respect to symmetry properties of molecular graph $\Gamma$. Many researchers have established, after diverse studies, a parallel between the physico chemical properties like boiling point, stability, similarity, chirality and melting point of chemical species and corresponding chemical graph. These descriptors defined on chemical graphs are extremely helpful for researchers to conduct regression model like QSAR/QSPR and better understand the physical features, complexity of molecules, chemical and biological properties of underlying compound.

In this paper, several structure descriptors of vital importance, namely, first, second, modified and augmented Zagreb indices, inverse and general Randic indices, symmetric division, harmonic, inverse sum and forgotten indices of Hex-derived Meshes (networks) of two kinds, namely, $H D N 1(n)$ and $H D N 2(n)$ are computed and recovered using general approach of topological polynomials.
\end{abstract}

Keywords: Topological indices; M-Polynomial; Forgotten Polynomial; Molecular graphs; Hex-derive networks

MSC: 5C12, $05 \mathrm{C} 90$

\footnotetext{
Xing-Long Wang: Department of General Education, Anhui Xinhua University, Hefei 230088, China, xinglong159wang@163.com Jia-Bao Liu: School of Mathematics and Physics, Anhui Jianzhu University, Hefei, 230601, P.R. China, E-mail: liujiabaoad@163.com Maqsood Ahmad: Department of Mathematics, COMSATS University Islamabad, Lahore Campus, 54000, Pakistan Department of Mathematics, University of Management and Technology, Lahore, 54000, Pakistan, E-mail:

mahmad@ciitlahore.edu.pk

Muhammad Hussain: Department of Mathematics, COMSATS University Islamabad, Lahore Campus, 54000, Pakistan, E-mail: mhmaths@gmail.com

Muhammad Saeed: Department of Mathematics, University of Management and Technology, Lahore, 54000, Pakistan, E-mail: mth.cod@umt.edu.pk
}

\section{Introduction}

Graph theory is a standout amongst the most extraordinary and one of a kind branch of mathematics by which the showing of any structure is made possible. As of late, it achieves much consideration among scientists on account of its extensive variety of utilizations in Computer science, electrical systems, interconnected systems, biological networks, and in chemistry, and so forth. The chemical graph theory is the quickly developing zone among scientists. It helps in comprehension about the basic properties of a molecular graph. There is a considerable measure of molecular compounds which have assortment of utilizations in the fields of business, commercial, industrial, pharmaceutical chemistry, every day life, and in research facility.

In recent years researchers got immense attraction toward an emerging field Cheminformatics, an interplay between Chemistry, Mathematics, Statistics and Information Science. In fact, one of the main reasons behind the significance of Cheminformatics is the interlacing of these core areas of science. Graph Theory attained exceptional place in Mathematical Chemistry and this novel branch got the name Chemical graph theory which became increasingly common among researchers and deals with molecular graph of a chemical compound to calculate various topological indices to understand and predict the physicochemical properties of chemical compounds [1]. During QSAR/ QSPR study, the regression analysis relies upon molecular descriptors and is responsible to understand and predict the chemical, biological and physical characteristics of compounds. This provides basis in designing new chemical compounds and drugs having features of our own interest.

In literature, numerous types of degree based, distance based as well as topological polynomials [2] related topo-

\footnotetext{
${ }^{\star}$ Corresponding Author: Muhammad Kamran Siddiqui: Department of Mathematics, COMSATS University Islamabad, Sahiwal Campus, 57000, Pakistan, E-mail: kamransiddiqui75@gmail.com
} 
logical indices of molecular graphs have been introduced and many of them turned out to be applicable in mathematical chemistry. For instance, among the most wellknown and well read graph invariants are the Wiener index, Szeged index [3], the Randić indices, Zagreb indices $[4,5]$, atom-bond connectivity, geometric arithmetic and the Hosoya $Z$ indices [6]. Although all above mentioned classes of indices have their own significance, however, degree based indices are well read and find real importance in chemical graph theory and therein biochemistry [7-11].

A graph $\Gamma(V, E)$ with vertex set $V$ and edge set $E$ is connected, if there exists a connection between any pair of vertices in $\Gamma$. A network is simply a connected graph having no multiple edges and loops. For a graph $\Gamma$, the degree of a vertex $v$ is the number of edges incident with $v$ and denoted by $d_{v}$.

Paul Manuel et al., [12] conjectured that the minimum metric dimension of hex-derived networks $H D N 1(n)$ and $H D N 2(n)$ lies between 3 and 5 and this open problem has partially been answered by Dacheng Xu and Jianxi Fan [13]. Furthermore, Imran et al.,[14] discussed some topological properties such as atom-bond connectivity, geometric arithmetic and Randić indices of the network under discussion. We have studied and computed some new indices as well as recovered some indices presented in [1519] by using entirely different and general approach.

In this article, throughout, $\Gamma$ is considered to be connected, finite, undirected and simple network with $V(\Gamma)=$ Vertex set, $E(\Gamma)=$ Edge set and $d_{v}=$ degree of vertex where $v \in V(\Gamma)$.

\subsection{Preliminaries}

Definition 1. Deutsch and Klavžar [20] introduced Mpolynomial for graph $\Gamma=(V, E)$ as follows:

$$
M(\Gamma ; x, y)=f(x, y)=\sum_{i \leq j} m_{i j}(\Gamma) x^{i} y^{j}
$$

where $m_{i j}(\Gamma)$ represent number of edges $u v \in E(\Gamma)$ such that $\left\{d_{u}(\Gamma), d_{v}(\Gamma)\right\}=\{i, j\}$.

Definition 2. The forgotten polynomial of $\Gamma$ is given by:

$$
F(\Gamma ; x)=\sum_{u v \in E(\Gamma)} x^{\left[\left(d_{u}\right)^{2}+\left(d_{v}\right)^{2}\right]}
$$

In 1947, the idea of topological index was first conceived and originated by Harold Wiener [21] during the study on boiling point of paraffin (bi-product of petroleum) and he referred it as path number but afterward path number was assigned the name of its inventor and entitled as Wiener index/number [22]. This pioneering, eminent and well studied index of chemical graph $\Gamma$ is distance-based index. It deserves high rank in theoretical Chemistry and Chemical graph theory due to its theoretical as well as applicable nature. In 1975, Milan Randić [23] introduced a topological index with the name branching index and is defined as:

$$
R_{\frac{-1}{2}}(\Gamma)=\sum_{u v \in E(\Gamma)} \frac{1}{\sqrt{d_{u} d_{v}}}
$$

Randic observed and established the fact that there exists a relationship between Randić index and various properties (boiling point, enthalpy of formation, surface area) of alkanes.

Later on in 1998, two distinguished researchers, Böllöbás and Erdös [24], extended the idea to all real numbers and the new index received the name general Randic $c$ index and is defined below:

$$
R_{\alpha}(\Gamma)=\sum_{u v \in E(\Gamma)} \frac{1}{\left(d_{u} d_{v}\right)^{\alpha}}
$$

Moreover, inverse Randić index is defined by formula,

$$
R R_{\alpha}(\Gamma)=\sum_{u v \in E(\Gamma)}\left(d_{u} d_{v}\right)^{\alpha}
$$

In 1972, Ivan Gutman and Trinajstic [25] proposed two topological indices named as the first and the second $\mathrm{Za}$ greb indices and soon after, these indices were used to analyze the structure-dependency of total $\pi$-electron of molecular graph. First, second and modified Zagreb indices are defined as follows:

$$
\begin{gathered}
M_{1}(\Gamma)=\sum_{v w \in E}\left(d_{v}+d_{w}\right) \\
M_{2}(\Gamma)=\sum_{v w \in E}\left(d_{v} d_{w}\right) \\
{ }^{m} M_{2}(\Gamma)=\sum_{\nu w \in E} \frac{1}{\left(d_{\nu} d_{w}\right)}
\end{gathered}
$$

\section{Definition 1. Generalized Zagreb Index}

The concept of generalized Zagreb index was established by Azari and Iranmanesh [26] and defined as

$$
Z_{a, b}(\Gamma)=\sum_{u v \in E(\Gamma)}\left(d_{u}^{a} d_{v}^{b}+d_{u}^{b} d_{v}^{a}\right)
$$

where $a, b \in Z^{+}$. Few more topological indices of our interest having utmost importance are defined below which include harmonic index (HI), inverse sum index (ISI), augmented Zagreb index (AZI) [27] and forgotten index (FI):

$$
H I(\Gamma)=\sum_{u v \in E(T)} \frac{2}{d_{u}+d_{v}}
$$




$$
\begin{gathered}
\operatorname{ISI}(\Gamma)=\sum_{u v \in E(\Gamma)} \frac{d_{u} d_{v}}{d_{u}+d_{v}} \\
A Z I(\Gamma)=\sum_{u v \in E(\Gamma)}\left(\frac{d_{u} d_{v}}{d_{u}+d_{v}-2}\right)^{3} \\
F I(\Gamma)=\sum_{u v \in E(\Gamma)}\left(d_{u}^{2}+d_{v}^{2}\right)
\end{gathered}
$$

\subsection{Applications of Topological polynomials}

Several topological polynomials were established in the literature and played vital role in mathematical chemistry. Among other graph polynomials like matching polynomial [28], the Zhang-Zhang polynomial (Clar covering polynomial) [29], the Schultz polynomial [30], the Tutte polynomial [31], the most significant polynomials are Hosoya polynomial [32] introduced in 1988 and $M$ polynomial established in 2015. We can efficiently determine exact formulae for various degree and distancebased topological indices with the help of M-polynomial and Hosoya polynomial, respectively. M-polynomial is the tool which conceals lot of facts regarding degree based graph invariants. Moreover, the $M$-polynomial provides a very good correlation for the stability of linear alkanes as well as the branched alkanes and for computing the strain energy of cyclo alkanes [33]. For a certain family of networks, we normally use different formula to calculate each individual topological index. M-polynomial got advantage over this approach as we only need to operate specific differential, integral or both operators on corresponding polynomial to get various vertex-based indices. Many closed form degree-based topological indices of Triangular Boron Nanotubes and Jahangir graph $J_{n, m}$ are computed using M-polynomial [34, 35]. The Zhang-Zhang polynomial (Clar covering polynomial), were found to occur for computation of the total $\pi$-electron energy of the molecules within specific approximate expressions. The Randic index is a topological descriptor that has correlated with a lot of chemical characteristics of the molecules and has been found to be parallel to compute the boiling point and Kovats constants of the molecules.

\section{Materials and Methods}

Our main results includes the formulation of algebraic structures of M-polynomial and F-polynomial of HDN1(n) and $H D N 2(n)$, respectively. Then, we computed as well as recovered various topological indices of vital importance. In particular, first, second, modified and augmented $\mathrm{Za}$ greb indices, general and inverse Randić indices, symmetric division, harmonic, inverse sum and forgotten indices of these networks via topological polynomials.

To compute our results, we used the method of combinatorial computing, vertex partition method, edge partition method, graph theoretical tools, analytic techniques, degree counting method, and sum of degrees of neighbor's method. Moreover, we used Maple for mathematical calculations, verifications, and plotting these mathematical results.

\section{Main Results}

\subsection{Methodology and Construction of Hex-Derived Network HDN1(n) Formulas}

The construction of first kind of hex-derived network $\operatorname{HDN1}(n)$ is fairly simple and is achieved by placing additional node in each triangular face of hexagonal mesh $H X(n)$ [36] and then joining this extra nodes with all nodes of triangular face. For an alternate version of construction of $H D N 1(n)$ from $H X(n)$. There are $9 n^{2}-15 n+7$ number of nodes (vertices) and $27 n^{2}-51 n+24$ number of edges in $H D N 1(n)$. This new network has many advantage over the one from which it is obtained, for instance, represent configuration similar to molecular lattice structures in chemistry. This network is also called mesh network and mostly used in networking of computers to minimize cost, to achieve high performance and reliability. Moreover, $H D N 1(n)$ is planar and this property gets advantage over non-planar network as far as cost is concerned. Figure 1 depicts a hex-derived network of first kind with dimension 4.

For the sake of simplicity as well as with out loss of generality, we assume $H D N 1(n)=\Gamma_{1}$. As we know total number of vertices of $\Gamma_{1}$ are given by $\left|V\left(\Gamma_{1}\right)\right|=9 n^{2}-15 n+7$ and total number of edges are $\left|E\left(\Gamma_{1}\right)\right|=27 n^{2}-51 n+24$. In $\Gamma_{1}$, we observe eight categories of edges on the basis of degree of the vertices of each edge which lead to edge partition of graph and is depicted in the table below.

Now, using definition 1 and definition 2 to compute $M$ Polynomial and Forgotten Polynomial, respectively of $\Gamma_{1}$ as follows:

- M-Polynomial of hex-derive network $H D N 1(n)$ 
Table 1: Formulae of some prominent topological descriptors depending on M-polynomial.

\begin{tabular}{cc}
\hline Topological Descriptors & Formulae based on M-polynomial \\
\hline 1st Zagreb index $\left(M_{1}\right)$ & $\left(D_{x}+D_{y}\right) f(x, y)$ \\
2nd Zagreb index $\left(M_{2}\right)$ & $\left(D_{x} \cdot D_{y}\right) f(x, y)$ \\
Modified 2nd Zagreb index $\left({ }^{m} M_{2}\right)$ & $\left(S_{x} \cdot S_{y}\right) f(x, y)$ \\
General Randić index $R_{\alpha}$ & $D_{x}^{\alpha} \cdot D_{y}^{\alpha} f(x, y)$ \\
Inverse Randić index $R R_{\alpha}$ & $S_{x}^{\alpha} \cdot S_{y}^{\alpha} f(x, y)$ \\
Symmetric Division Index (SDD) & $\left(D_{x} S_{y}+S_{x} D_{y}\right) f(x, y)$ \\
Harmonic Index (HI) & $2 S_{x} J f(x, y)$ \\
Inverse sum Index (ISI) & $S_{x} J D_{x} D_{y} f(x, y)$ \\
Augmented Zagreb Index (AG) & $S_{x}^{3} Q_{-2} J D_{x}^{3} D_{y}^{3} f(x, y)$ \\
Forgotten Index(FI) & $D_{x},[f(G ; x)], x=1$
\end{tabular}

where $D_{x} f=x \frac{\partial f}{\partial x}, D_{y} f=y \frac{\partial f}{\partial y}, S_{x} f=\int_{0}^{x} \frac{f(t, y)}{t} d t, S_{y} f=\int_{0}^{y} \frac{f(x, t)}{t} d t, J(f(x, y))=f(x, x), Q_{\alpha} f=x^{\alpha} f$.

Table 2: Vertex degree based edge partitioning of a graph $\Gamma_{1}$.

\begin{tabular}{ccccccccc}
\hline $\begin{array}{c}\left(d_{u}, d_{v}\right) \\
u v \in E\left(\Gamma_{1}\right)\end{array}$ & $(3,5)$ & $(3,7)$ & $(3,12)$ & $(5,7)$ & $(5,12)$ & $(7,7)$ & $(7,12)$ & $(12,12)$ \\
\hline $\begin{array}{c}\text { Number } \\
\text { of edges }\end{array}$ & 12 & $18 n-36$ & $18 n^{2}-54 n+42$ & 12 & 6 & $6 n-18$ & $12 n-24$ & $9 n^{2}-33 n+30$ \\
\hline $\begin{array}{c}\text { Set } \\
\text { of edges }\end{array}$ & $E_{1}$ & $E_{2}$ & $E_{3}$ & $E_{4}$ & $E_{5}$ & $E_{6}$ & $E_{7}$ & $E_{8}$ \\
\hline
\end{tabular}




$$
\begin{aligned}
M\left(\Gamma_{1} ; x, y\right) & =\sum_{i \leq j} m_{i j} x^{i} y^{j} \\
& =\sum_{3 \leq 5} m_{35} x^{3} y^{5}+\sum_{3 \leq 7} m_{37} x^{3} y^{7}+\sum_{3 \leq 12} m_{312} x^{3} y^{12}+\sum_{5 \leq 7} m_{57} x^{5} y^{7} \\
& +\sum_{5 \leq 12} m_{512} x^{5} y^{12}+\sum_{7 \leq 7} m_{77} x^{7} y^{7}+\sum_{7 \leq 12} m_{712} x^{7} y^{12}+\sum_{12 \leq 12} m_{1212} x^{12} y^{12} \\
& =\sum_{u v \in E_{1}} m_{35} x^{3} y^{5}+\sum_{u v \in E_{2}} m_{37} x^{3} y^{7}+\sum_{u v \in E_{3}} m_{312} x^{3} y^{12}+\sum_{u v \in E_{4}} m_{57} x^{5} y^{7} \\
& +\sum_{u v \in E_{5}} m_{512} x^{5} y^{12}+\sum_{u v \in E_{6}} m_{77} x^{7} y^{7}+\sum_{u v \in E_{7}} m_{712} x^{7} y^{12}+\sum_{u v \in E_{8}} m_{1212} x^{12} y^{12} \\
& =\left|E_{1}\right| x^{3} y^{5}+\left|E_{2}\right| x^{3} y^{7}+\left|E_{3}\right| x^{3} y^{12}+\left|E_{4}\right| x^{5} y^{7} \\
& +\left|E_{5}\right| x^{5} y^{12}+\left|E_{6}\right| x^{7} y^{7}+\left|E_{7}\right| x^{7} y^{12}+\left|E_{8}\right| x^{12} y^{12} \\
& =12 x^{3} y^{5}+(18 n-36) x^{3} y^{7}+\left(18 n^{2}-54 n+42\right) x^{3} y^{12}+(6 n-18) x^{7} y^{7} \\
& +12 x^{5} y^{7}+6 x^{5} y^{12}+(12 n-24) x^{7} y^{12}+\left(9 n^{2}-33 n+30\right) x^{12} y^{12}
\end{aligned}
$$

Following Figure 2 depicts graphs of M-polynomial of hex-derive network HDN1(4).

- Forgotten Polynomial of hex-derive network $H D N 1(n)$

$$
\begin{aligned}
F\left(\Gamma_{1}, x\right) & =\sum_{u v \in E(G)} x^{\left[\left(d_{u}\right)^{2}+\left(d_{v}\right)^{2}\right]} \\
& =\sum_{u v \in E_{1}} m_{35} x^{34}+\sum_{u v \in E_{2}} m_{37} x^{58}+\sum_{u v \in E_{3}} m_{312} x^{153}+\sum_{u v \in E_{4}} m_{57} x^{74} \\
& +\sum_{u v \in E_{5}} m_{512} x^{169}+\sum_{u v \in E_{6}} m_{77} x^{98}+\sum_{u v \in E_{7}} m_{712} x^{193}+\sum_{u v \in E_{8}} m_{1212} x^{288} \\
& =12 x^{34}+(18 n-36) x^{58}+\left(18 n^{2}-54 n+42\right) x^{153}+12 x^{74}+6 x^{169} \\
& +(6 n-18) x^{98}+(12 n-24) x^{193}+\left(9 n^{2}-33 n+30\right) x^{288}
\end{aligned}
$$

Following Figure 3 depicts graphs of Forgotten polynomial of hex-derive network HDN1(4).

- Computing Topological Indices using M-polynomial and Forgotten polynomial for hex-derive network $\operatorname{HDN} 1(n)$

Now we compute the toplogical indices for hex-derive network $\Gamma_{1}$, namely first, second, modified and augmented Zagreb indices, Randić indices, SSD index, harmonic index, ISI index and forgotten index. By applying the operators given in derivation of Table 1 on M-polynomial and Forgotten polynomials as follows:

$$
\begin{aligned}
\left(D_{x}+D_{y}\right) f(x, y)= & 10(18 n-36) x^{3} y^{7}+15\left(18 n^{2}-54 n+42\right) x^{3} y^{12}+96 x^{3} y^{5}+144 x^{5} y^{7}+102 x^{5} y^{12} \\
+ & 14(6 n-18) x^{7} y^{7}+19(12 n-24) x^{7} y^{12}+24\left(9 n^{2}-33 n+30\right) x^{12} y^{12} \\
D_{x}^{\alpha} D_{y}^{\alpha} f(x, y)= & 21^{\alpha}(18 n-36) x^{3} y^{7}+36^{\alpha}\left(18 n^{2}-54 n+42\right) x^{3} y^{12}+15^{\alpha}\left(12 x^{3} y^{5}\right) \\
+ & 35^{\alpha}\left(12 x^{5} y^{7}\right)+60^{\alpha}\left(6 x^{5} y^{12}\right)+84^{\alpha}(12 n-24) x^{7} y^{12} \\
+ & 7^{2 \alpha}(6 n-18) x^{7} y^{7}+12^{2 \alpha}\left(9 n^{2}-33 n+30\right) x^{12} y^{12}
\end{aligned}
$$




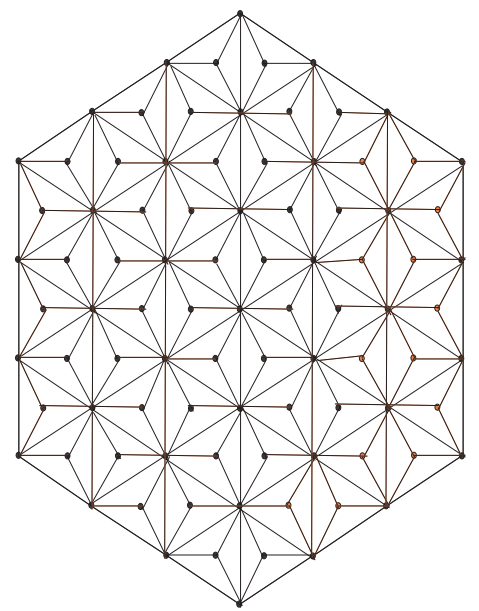

Figure 1: A 4-dimensional hex-derive network $H D N 1(4)$

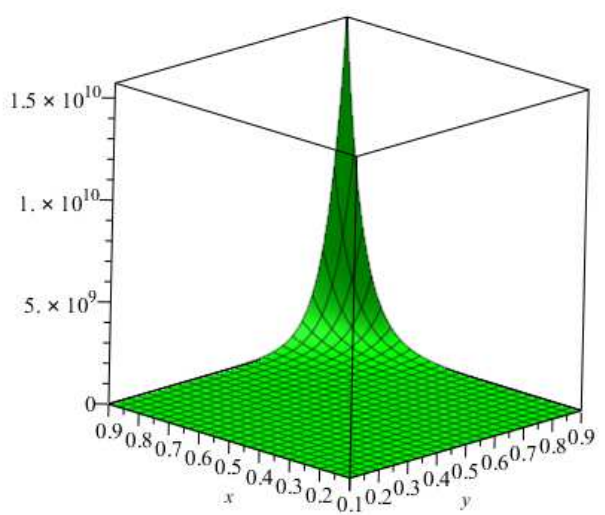

Figure 2: Graphical Representation of M-polynomial of hex-derive network $\operatorname{HDN} 1(n)$

$$
\begin{aligned}
S_{x}^{\alpha} S_{y}^{\alpha} f(x, y)= & \frac{1}{21^{\alpha}}(18 n-36) x^{3} y^{7}+\frac{1}{36^{\alpha}}\left(18 n^{2}-54 n+42\right) x^{3} y^{12}+\frac{12}{15^{\alpha}} x^{3} y^{5} \\
+ & \frac{12}{35^{\alpha}} x^{5} y^{7}+\frac{6}{60^{\alpha}} x^{5} y^{12}+\frac{1}{7^{2 \alpha}}(6 n-18) x^{7} y^{7} \\
+ & \frac{1}{84^{\alpha}}(12 n-24) x^{7} y^{12}+\frac{1}{12^{2 \alpha}}\left(9 n^{2}-33 n+30\right) x^{12} y^{12} \\
\left(S_{y} D_{x}+S_{x} D_{y}\right) f(x, y)= & \frac{58}{21}(18 n-36) x^{3} y^{7}+\frac{17}{4}\left(18 n^{2}-54 n+42\right) x^{3} y^{12} \\
& +\frac{888}{35} x^{5} y^{7}+\frac{169}{10} x^{5} y^{12}+2(6 n-18) x^{7} y^{7}+\frac{193}{84}(12 n-24) x^{7} y^{12} \\
& +\frac{136}{5} x^{3} y^{5}+2\left(9 n^{2}-33 n+30\right) x^{12} y^{12} \\
= & 12 x^{8}+(18 n-36) x^{10}+\left(18 n^{2}-54 n+42\right) x^{15} \\
+ & 12 x^{12}+6 x^{17}+(6 n-18) x^{14}+(12 n-24) x^{19}+\left(9 n^{2}-33 n+30\right) x^{24}
\end{aligned}
$$




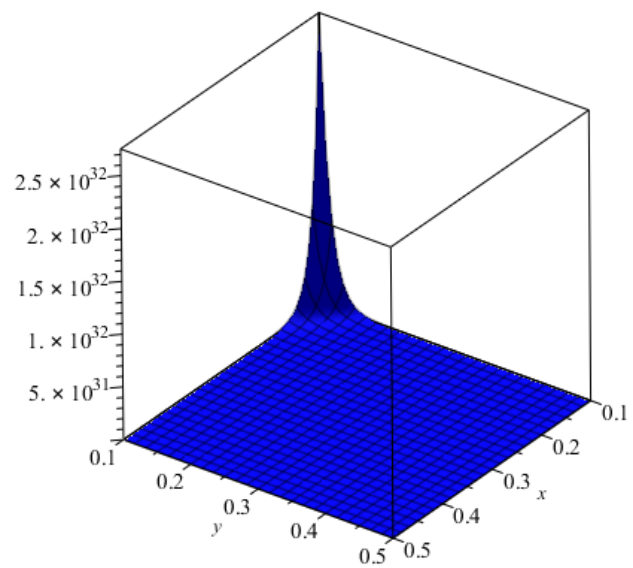

Figure 3: Graphical Representation of Forgotten polynomial of hex-derive network $\operatorname{HDN} 1(n)$

$$
\begin{aligned}
S_{x} J D_{x} D_{y} f(x, y)= & \frac{45}{2} x^{8}+\frac{1}{5}(18 n-36) x^{10}+\frac{12}{5}\left(18 n^{2}-54 n+42\right) x^{15} \\
+ & 35 x^{12}+\frac{360}{17} x^{17}+\frac{7}{2}(6 n-18) x^{14}+\frac{84}{19}(12 n-24) x^{19}+6\left(9 n^{2}-33 n+30\right) x^{24} \\
S_{x}^{3} Q_{-2} J D_{x}^{3} D_{y}^{3} f(x, y)= & \frac{375}{2} x^{6}+\frac{46656}{2197}\left(18 n^{2}-54 n+42\right) x^{13} \\
& +\frac{9261}{512}(18 n-36) x^{8}+\frac{1029}{2} x^{10}+384 x^{15}+\frac{117649}{1728}(6 n-18) x^{12} \\
& +\frac{592704}{4913}(12 n-24) x^{17}+\frac{373248}{1331}\left(9 n^{2}-33 n+30\right) x^{22}
\end{aligned}
$$

Again using derivation formulae of topological indices over M-polynomial from Table 1, we get

$$
\begin{aligned}
\text { FirtsZagrebIndex }=M_{1}\left(\Gamma_{1}\right) & =\left.\left(D_{x}+D_{y}\right) f(x, y)\right|_{x=y=1} \\
& =486 n^{2}-1110 n+624, \\
\text { SecondZagrebIndex }=M_{2}\left(\Gamma_{1}\right) & =\left.D_{y} D_{x} f(x, y)\right|_{x=y=1} \\
& =1944 n^{2}-5016 n-750 \\
\text { ModifiedZagrebIndex }{ }^{m}{ }^{m} M_{2}\left(\Gamma_{1}\right) & =\left.S_{x} S_{y} f(x, y)\right|_{x=y=1} \\
& =\frac{9}{16} n^{2}-\frac{7475}{2352} n+\frac{491}{1960} \\
\text { Generalized Randić Index }=R_{\alpha}\left(\Gamma_{1}\right) & =\left.D_{x}^{\alpha} D_{y}^{\alpha} f(x, y)\right|_{x=y=1} \\
& =12\left(15^{\alpha}+35^{\alpha}\right)+6\left(60^{\alpha}\right)+21^{\alpha}(18 n-36) \\
& +36^{\alpha}\left(18 n^{2}-54 n+42\right)+7^{2 \alpha}(6 n-18) \\
& +84^{\alpha}(12 n-24)+12^{2 \alpha}\left(9 n^{2}-33 n+30\right)
\end{aligned}
$$




$$
\begin{aligned}
\text { Inverse Randić Index }=R R_{\alpha}\left(\Gamma_{1}\right)= & \left.S_{x}^{\alpha} S_{y}^{\alpha} f(x, y)\right|_{x=y=1} \\
= & 12\left(15^{-\alpha}+35^{-\alpha}\right)+6\left(60^{-\alpha}\right)+36^{-\alpha}\left(18 n^{2}-54 n+42\right) \\
& +21^{-\alpha}(18 n-36)+7^{-2 \alpha}(6 n-18)+84^{-\alpha}(12 n-24) \\
& +12^{-2 \alpha}\left(9 n^{2}-33 n+30\right) \\
\text { Symmetric Division Index }=S S D\left(\Gamma_{1}\right)= & \left.\left(D_{x} S_{y}+S_{x} D_{y}\right) f(x, y)\right|_{x=y=1} \\
= & \frac{189}{2} n^{2}-\frac{2887}{14} n+\frac{587}{5} \\
\text { Harmonic Index }=H I\left(\Gamma_{1}\right)= & \left.2 S_{x} J f(x, x)\right|_{x=1} \\
= & \frac{63}{20} n^{2}-\frac{11251}{2660} n+\frac{34099}{22610} \\
\text { Inverse Sum Index }=\operatorname{ISI}\left(\Gamma_{1}\right)= & \left.S_{x} J D_{x} D_{y} f(x, y)\right|_{x=y=1} \\
= & \frac{486}{5} n^{2}-\frac{648}{323} n+\frac{627043}{3230} \\
\text { Forgotten Index }=F\left(\Gamma_{1}\right) \quad= & \left.D_{x} F\left(\Gamma_{1}, x\right)\right|_{x=1}=5346 n^{2}-13818 n+8892 . \\
& =2906 n^{2}-8219 n+5619
\end{aligned}
$$

\subsection{Methodology and Construction of Hex-Derived Network $\operatorname{HDN2}(n)$ Formulas}

The architecture of second kind of hex-derived network $H D N 2(n)$ is bit sophisticated as it is obtained from the merger of hexagonal network $H X(n)$ of dimension $n$ with honeycomb network $H C(n-1)$ of dimension $n-1$. The construction of $H D N 2(n)$ can be accomplished by taking union of $H X(n)$ with its bounded dual $H C(n-1)$ and then by joining each honeycomb vertex with the three vertices of the corresponding face of $H X(n)$. There are $9 n^{2}-15 n+7$ number of nodes (vertices) and $36 n^{2}-72 n+36$ number of edges in $H D N 2(n)$. Figure 4 depicts a hex-derived network of second kind with dimension 4.

Again for the sake of simplicity, suppose $\operatorname{HDN} 2(n)=\Gamma_{2}$. We know total number of vertices of $\Gamma_{2}$ are given by $\left|V\left(\Gamma_{2}\right)\right|=9 n^{2}-15 n+7$ and total number of edges are $\left|E\left(\Gamma_{2}\right)\right|=36 n^{2}-72 n+36$. In $\Gamma_{2}$, we observe eight categories of edges on the basis of degree of the vertices of each edge which lead to edge partition of graph and is depicted in the table below. Now, using definition 1 and definition 2 to compute M-Polynomial and Forgotten Polynomial, respectively

Table 3: Vertex degree based edge partitioning of a graph $\Gamma_{2}$.

\begin{tabular}{cccccc}
\hline$\left(d_{u}, d_{v}\right): u v \in E\left(\Gamma_{2}\right)$ & Number of edges & Sets & $\left(d_{u}, d_{v}\right): u v \in E\left(\Gamma_{2}\right)$ & Number of edges & Sets \\
\hline$(5,5)$ & 18 & $E_{1}$ & $(6,7)$ & $6(n-2)$ & $E_{6}$ \\
$(5,6)$ & $12(n-2)$ & $E_{2}$ & $(6,12)$ & $6\left(3 n^{2}-10 n+8\right)$ & $E_{7}$ \\
$(5,7)$ & $12(n-1)$ & $E_{3}$ & $(7,7)$ & $6(n-3)$ & $E_{8}$ \\
$(5,12)$ & $6 \mathrm{n}$ & $E_{4}$ & $(7,12)$ & $12(n-2)$ & $E_{9}$ \\
$(6,6)$ & $3\left(3 n^{2}-11 n+10\right)$ & $E_{5}$ & $(12,12)$ & $3\left(3 n^{2}-11 n+10\right)$ & $E_{10}$
\end{tabular}

of $\Gamma_{2}$ as follows: 


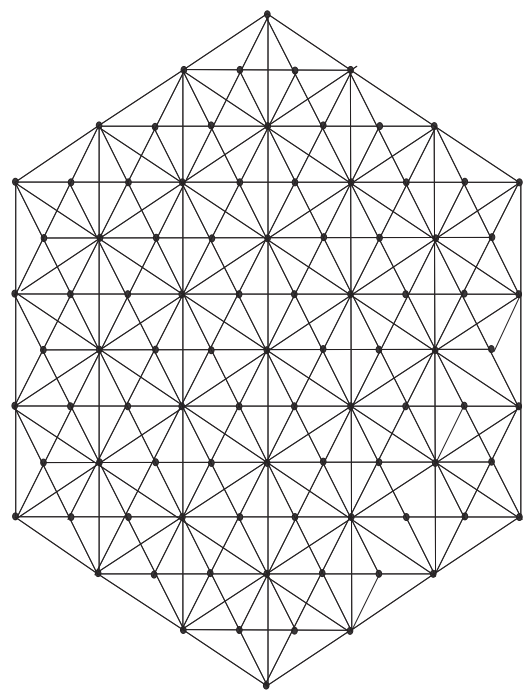

Figure 4: A 4-dimensional hex-derive network $H D N 2(4)$

\section{- M-Polynomial of hex-derive network $H D N 2(n)$}

$$
\begin{aligned}
M\left(\Gamma_{2} ; x, y\right)= & \sum_{i \leq j} m_{i j} x^{i} y^{j} \\
& =\sum_{5 \leq 5} m_{55} x^{5} y^{5}+\sum_{5 \leq 6} m_{56} x^{5} y^{6}+\sum_{5 \leq 7} m_{57} x^{5} y^{7}+\sum_{5 \leq 12} m_{512} x^{5} y^{12} \\
& +\sum_{6 \leq 6} m_{66} x^{6} y^{6}+\sum_{6 \leq 7} m_{67} x^{6} y^{7}+\sum_{6 \leq 12} m_{612} x^{6} y^{12}+\sum_{7 \leq 7} m_{77} x^{7} y^{7} \\
& +\sum_{7 \leq 12} m_{712} x^{7} y^{12}+\sum_{12 \leq 12} m_{1212} x^{12} y^{12} \\
M\left(\Gamma_{2} ; x, y\right)= & \sum_{u v \in E_{1}} m_{55} x^{5} y^{5}+\sum_{u v \in E_{2}} m_{56} x^{5} y^{6}+\sum_{u v \in E_{3}} m_{57} x^{5} y^{7}+\sum_{u v \in E_{4}} m_{512} x^{5} y^{12} \\
+ & \sum_{u v \in E_{5}} m_{66} x^{6} y^{6}+\sum_{u v \in E_{6}} m_{67} x^{6} y^{7}+\sum_{u v \in E_{7}} m_{612} x^{6} y^{12}+\sum_{u v \in E_{8}} m_{77} x^{7} y^{7} \\
+ & \sum_{u v \in E_{9}} m_{712} x^{7} y^{12}+\sum_{u v \in E_{10}} m_{1212} x^{12} y^{12} \\
= & \left|E_{1}\right| x^{5} y^{5}+\left|E_{2}\right| x^{5} y^{6}+\left|E_{3}\right| x^{5} y^{7}+\left|E_{4}\right| x^{5} y^{12}+\left|E_{5}\right| x^{6} y^{6} \\
+ & \left|E_{6}\right| x^{6} y^{7}+\left|E_{7}\right| x^{6} y^{12}+\left|E_{8}\right| x^{7} y^{7}+\left|E_{9}\right| x^{7} y^{12}+\left|E_{10}\right| x^{12} y^{12} \\
= & 18 x^{5} y^{5}+12(n-2) x^{5} y^{6}+12(n-1) x^{5} y^{7}+3\left(3 n^{2}\right. \\
& -11 n+10) x^{6} y^{6}+6 n x^{5} y^{12}+6(n-2) x^{6} y^{7}+6\left(3 n^{2}-10 n+8\right) x^{6} y^{12} \\
+ & 6(n-3) x^{7} y^{7}+12(n-2) x^{7} y^{12}+3\left(3 n^{2}-11 n+10\right) x^{12} y^{12}
\end{aligned}
$$

Following Figure 5 depicts graphs of M-polynomial of hex-derive network HDN2(4). 


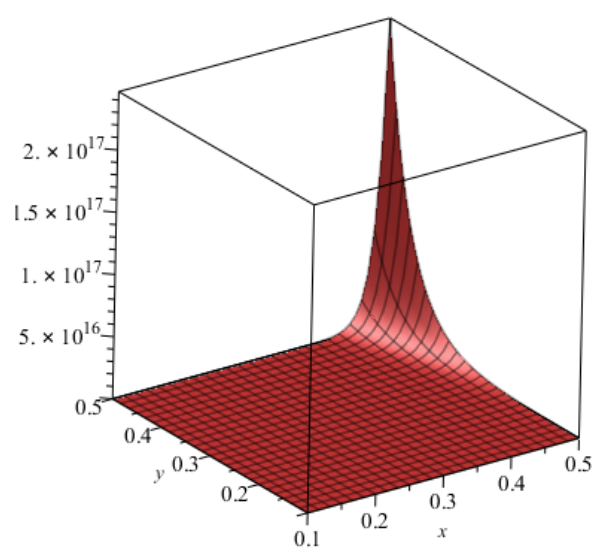

Figure 5: Graphical Representation of M-polynomial of hex-derive network HDN2(n)

- Forgotten Polynomial of hex-derive network $H D N 2(n)$

$$
\begin{aligned}
F\left(\Gamma_{2}, x\right) & =\sum_{u v \in E(G)} x^{\left[\left(d_{u}\right)^{2}+\left(d_{v}\right)^{2}\right]} \\
& =\sum_{u v \in E_{1}} m_{55} x^{50}+\sum_{u v \in E_{2}} m_{56} x^{61}+\sum_{u v \in E_{3}} m_{57} x^{74}+\sum_{u v \in E_{4}} m_{512} x^{169}+\sum_{u v \in E_{5}} m_{66} x^{72} \\
& +\sum_{u v \in E_{6}} m_{67} x^{85}+\sum_{u v \in E_{7}} m_{612} x^{180}+\sum_{u v \in E_{8}} m_{77} x^{98}+\sum_{u v \in E_{9}} m_{712} x^{193}+\sum_{u v \in E_{10}} m_{1212} x^{288} \\
& =18 x^{50}+12(n-2) x^{61}+12(n-1) x^{74}+6 n x^{169}+3\left(3 n^{2}-11 n+10\right) x^{72} \\
& +6(n-2) x^{85}+6\left(3 n^{2}-10 n+8\right) x^{180}+6(n-3) x^{98} \\
& +12(n-2) x^{193}+3\left(3 n^{2}-11 n+10\right) x^{288}
\end{aligned}
$$

Following Figure 6 depicts graphs of Forgotten polynomial of hex-derive network HDN2(4).

- Computing Topological Indices using M-polynomial and Forgotten polynomial for hex-derive network $\operatorname{HDN} 2(n)$

Now we compute the toplogical indices for hex-derive network $\Gamma_{2}$, namely first, second, modified and augmented Zagreb indices, Randić indices, SSD index, harmonic index, ISI index and forgotten index. By applying the operators given in derivation of Table 1 on M-polynomial and Forgotten polynomials as follows:

$$
\begin{aligned}
M\left(\Gamma_{2} ; x, y\right) & =f(x, y) \\
& =12 x^{3} y^{5}+(18 n-36) x^{3} y^{7}+\left(18 n^{2}-54 n+42\right) x^{3} y^{12} \\
& +12 x^{5} y^{7}+6 x^{5} y^{12}+(6 n-18) x^{7} y^{7}+(12 n-24) x^{7} y^{12} \\
& +\left(9 n^{2}-33 n+30\right) x^{12} y^{12}
\end{aligned}
$$




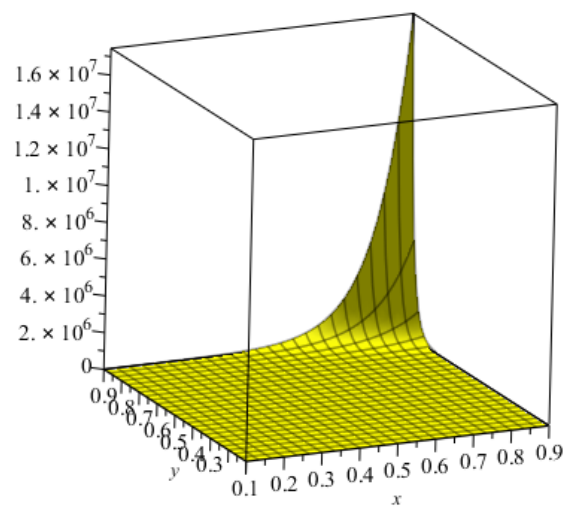

Figure 6: Graphical Representation of Forgotten polynomial of hex-derive network $\operatorname{HDN} 2(n)$

$$
\begin{aligned}
& \left(D_{x}+D_{y}\right) f(x, y)=180 x^{5} y^{5}+132(n-2) x^{5} y^{6}+144(n-1) x^{5} y^{7}+102 n x^{5} y^{12} \\
& +36\left(3 n^{2}-11 n+10\right) x^{6} y^{6}+78(n-2) x^{6} y^{7}+108\left(3 n^{2}-10 n+8\right) x^{6} y^{12} \\
& +84(n-2) x^{7} y^{12}+228(n-2) x^{7} y^{12}+72\left(3 n^{2}-11 n+10\right) x^{12} y^{12} \\
& D_{x}^{\alpha} D_{y}^{\alpha} f(x, y)=18\left(5^{2 \alpha}\right) x^{5} y^{5}+30^{\alpha}(12 n-24) x^{5} y^{6}+35^{\alpha}(12 n-12) x^{5} y^{7} \\
& +60^{\alpha}\left(6 n x^{5} y^{12}\right)+42^{\alpha}(6 n-12) x^{6} y^{7}+6^{2 \alpha}\left(9 n^{2}-33 n+30\right) x^{6} y^{6} \\
& +6^{1+\alpha} 12^{\alpha}\left(3 n^{2}-10 n+8\right) x^{6} y^{12}+7^{2 \alpha}(6 n-18) x^{7} y^{7}+12^{1+\alpha} 7^{\alpha}(n-2) x^{7} y^{12} \\
& +3.12^{2 \alpha}\left(3 n^{2}-11 n+10\right) x^{12} y^{12} \\
& \begin{aligned}
S_{x}^{\alpha} S_{y}^{\alpha} f(x, y) & =\left(\frac{1}{6^{2 \alpha}}+\frac{x^{6} y^{6}}{12^{2 \alpha}}\right)\left(9 n^{2}-33 n+30\right) x^{6} y^{6}+\frac{1}{72^{\alpha}}\left(18 n^{2}-60 n+48\right) x^{6} y^{12} \\
& +\frac{1}{35^{\alpha}}(12 n-12) x^{5} y^{7}+\left(\frac{2}{30^{\alpha}}+\frac{x y}{42^{\alpha}}+\frac{2 x^{6} y^{6}}{84^{\alpha}}\right)(6 n-12) x^{5} y^{6}+\frac{18}{5^{2 \alpha}} x^{5} y^{5} \\
& +\frac{6 n}{60^{\alpha}} x^{5} y^{12}+7^{-2 \alpha}(6 n-18) x^{7} y^{7}
\end{aligned} \\
& J f=f(x, x)=18 x^{10}+12(n-2) x^{11}+12(n-1) x^{12}+6 n x^{17}+6(n-3) x^{14} \\
& +3\left(3 n^{2}-11 n+10\right) x^{12}+6(n-2) x^{13}+6\left(3 n^{2}-10 n+8\right) x^{18} \\
& +12(n-2) x^{19}+3\left(3 n^{2}-11 n+10\right) x^{24} \\
& S_{x} J D_{x} D_{y} f(x, y)=45 x^{10}+\frac{30}{11}(n-2) x^{11}+35(n-1) x^{12} \\
& +\left(27 n^{2}-99 n+90\right) x^{12}\left(1+2 x^{12}\right)+\frac{252}{13}(n-2) x^{13}+\frac{360}{17} n x^{17} \\
& +24\left(3 n^{2}-10 n+8\right) x^{18}+21(n-3) x^{14}+\frac{1008}{19}(n-2) x^{19}
\end{aligned}
$$




$$
\begin{aligned}
S_{x}^{3} Q_{-2} J D_{x}^{3} D_{y}^{3} f(x, y) & =\frac{140625}{256} x^{8}+\left(\frac{4000}{9}+\frac{444528 x^{2}}{1331}+\frac{7112448 x^{8}}{4913}\right)(n-2) x^{9} \\
& +\frac{1029}{2} x^{10}+\left(\frac{17496}{125}+\frac{4478976 x^{12}}{5324}\right)\left(3 n^{2}-11 n+10\right) x^{10} \\
& +\frac{117649}{288}(n-3) x^{12}+384 n x^{15}+\frac{2187}{4}\left(3 n^{2}-10 n+8\right) x^{16}
\end{aligned}
$$

Again using derivation formulae of topological indices over M-polynomial from table 1, we get

$$
\begin{aligned}
& \text { FirstZagrebIndex }=M_{1}\left(\Gamma_{2}\right)=\left.\left(D_{x}+D_{y}\right) f(x, y)\right|_{x=y=1} \\
& =648 n^{2}-1500 n+852 \text {, } \\
& \text { Secondnd Zagreb Index }=M_{2}\left(\Gamma_{2}\right)=\left.D_{y} D_{x} f(x, y)\right|_{x=y=1} \\
& =2916 n^{2}-7566 n+4764, \\
& \text { Modified Zagreb Index }={ }^{m} M_{2}\left(\Gamma_{2}\right)=\left.S_{x} S_{y} f(x, y)\right|_{x=y=1} \\
& =\frac{9}{16} n^{2}-\frac{8563}{11760} n+\frac{30597}{88200} \\
& \text { Generalized Randić Index }=R_{\alpha}\left(\Gamma_{2}\right)=\left.D_{x}^{\alpha} D_{y}^{\alpha} f(x, y)\right|_{x=y=1} \\
& =6^{2 \alpha}\left(1+4^{\alpha}\right)\left(9 n^{2}-33 n+30\right)+6^{\alpha}\left(2.5^{\alpha}+60^{\alpha}(6 n)\right. \\
& \left.+\left(6^{1+2 \alpha} \cdot 2^{\alpha}\right)\left(3 n^{2}-10 n+8\right)+7^{\alpha}+2^{1+\alpha}\right)(6 n-12) \\
& +35^{\alpha}(12 n-12)+7^{2 \alpha}(6 n-18)+18\left(5^{2 \alpha}\right) \\
& \text { Inverse Randić Index }=R R_{\alpha}\left(\Gamma_{2}\right)=\left.S_{x}^{\alpha} S_{y}^{\alpha} f(x, y)\right|_{x=y=1} \\
& =12\left(15^{-\alpha}+35^{-\alpha}\right)+6\left(60^{-\alpha}\right)+21^{-\alpha}(18 n-36) \\
& +7^{-2 \alpha}(6 n-18)+84^{-\alpha}(12 n-24)+12^{-2 \alpha}\left(9 n^{2}-33 n+30\right) \\
& +36^{-\alpha}\left(18 n^{2}-54 n+42\right) \\
& =81 n^{2}-\frac{11453}{70} n+\frac{432}{5} \\
& \text { Harmonic Index }=H I\left(\Gamma_{2}\right)=\left.2 S_{x} J f(x, x)\right|_{x=1} \\
& =\frac{17}{4} n^{2}-\frac{27103217}{3879876} n+\frac{15629}{5000} \\
& \text { Inverse Sum Index }=\operatorname{ISI}\left(\Gamma_{2}\right)=\left.S_{x} J D_{x} D_{y} f(x, y)\right|_{x=y=1} \\
& =153 n^{2}-385 n-101 \\
& =4584 n^{2}-13243 n+9573
\end{aligned}
$$




\section{Comparisons and Discussion}

In this section, we have computed all indices for different values of $n$ for both structures $\operatorname{HDN1}(n)$ and $\operatorname{HDN} 2(n)$. In addition, we construct Tables 4 and 5 for small values of $n$ for these topological indices to the structure $H D N 1(n)$ and $H D N 2(n)$ respectively. Now, from Tables 4 and 5, we can easily see that all indices are in increasing order as the values of $n$ are increases. In addition, on the other hand, indices showed higher values for $H D N 2(n)$ as compared to those of $\operatorname{HDN} 1(n)$.

Table 4: Comparison of all indices for $\operatorname{HDN1}(n)$.

\begin{tabular}{ccccccccccc}
\hline $\boldsymbol{n}$ & $\boldsymbol{M}_{\mathbf{1}}$ & $\boldsymbol{M}_{\mathbf{2}}$ & $\boldsymbol{M}_{\mathbf{2}}^{\boldsymbol{m}}$ & $\boldsymbol{R}_{\boldsymbol{\alpha}}$ & $\boldsymbol{R}_{\boldsymbol{\alpha} \boldsymbol{\alpha}}$ & $\boldsymbol{S S D}$ & $\boldsymbol{H I}$ & $\boldsymbol{I S I}$ & $\boldsymbol{A Z I}$ & $\boldsymbol{F I}$ \\
\hline 1 & 0 & -3822 & 1.4 & 48.2 & 56.3 & 96.8 & 4.5 & 98.6 & 306 & 420 \\
2 & 348 & -3006 & 3.4 & 512.4 & 617.8 & 415.6 & 8.3 & 187.2 & 805 & 2640 \\
3 & 1668 & 1698 & 6.3 & 1021.1 & 1108.4 & 918.2 & 13.6 & 415.3 & 7116 & 15552 \\
4 & 3960 & 10290 & 9.7 & 1826.3 & 1936.5 & 1562.4 & 18.2 & 916.4 & 19239 & 39156 \\
5 & 7224 & 22770 & 12.8 & 2445.1 & 2916.4 & 2113.5 & 25.6 & 1023.6 & 37174 & 73452 \\
\hline
\end{tabular}

Table 5: Comparison of all indices for $\operatorname{HDN} 2(n)$.

\begin{tabular}{ccccccccccc}
\hline $\boldsymbol{n}$ & $\boldsymbol{M}_{\mathbf{1}}$ & $\boldsymbol{M}_{\mathbf{2}}$ & $\boldsymbol{M}_{\mathbf{2}}^{\boldsymbol{m}}$ & $\boldsymbol{R}_{\boldsymbol{\alpha}}$ & $\boldsymbol{R}_{\boldsymbol{\alpha} \boldsymbol{\alpha}}$ & $\boldsymbol{S S D}$ & $\boldsymbol{H I}$ & $\boldsymbol{I S I}$ & $\boldsymbol{A Z I}$ & $\boldsymbol{F I}$ \\
\hline 1 & 0 & 114 & 3.4 & 54.3 & 51.4 & 99.2 & 6.6 & 102 & 914 & 445 \\
2 & 444 & 1296 & 5.4 & 615.6 & 827.8 & 635.4 & 9.6 & 235 & 1423 & 2740 \\
3 & 2184 & 8310 & 9.3 & 1321.9 & 1508.2 & 1102.3 & 16.5 & 615 & 11100 & 16552 \\
4 & 5220 & 21156 & 13.7 & 1926.5 & 2136.4 & 1862.3 & 21.3 & 1002 & 29945 & 40156 \\
5 & 9552 & 39834 & 16.8 & 2645.2 & 3216.3 & 2313.6 & 29.3 & 1124 & 57959 & 75452 \\
\hline
\end{tabular}

Now, we presented the comparison of all topological indices using Table 4, for $H D N 1(n)$ in Figure 7 and using Table 5, for $H D N 2(n)$ in Figure 8.

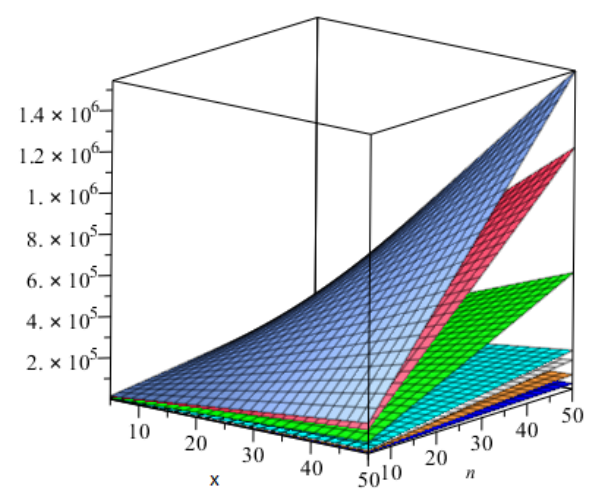

Figure 7: The comparison of all topological indices for $H D N 1(n)$. 


\section{Conclusions}

In this paper, we provide M-polynomials of two interesting networks $H D N 1(n)$ and $H D N 2(n)$. In addition, we offer closed form formulae of several degree-based topological indices of vital importance such as first, second, modified and augmented Zagreb indices, general and inverse Randić indices, SSD, HI, ISI and forgotten index of $H D N 1(n)$ and $H D N 2(n)$ are computed and recovered using topological polynomials attained in previous step.

Since the Randić index is a topological descriptor that has correlated with a lot of chemical characteristics of the molecules. Thus, it has been found that the boiling point of $H D N 1(n)$ and $H D N 2(n)$ is varying in increasing order for $\alpha \in\{1,-1,1 / 2,-1 / 2\}$.

Since the SSD index and HI index provides a very good correlation for computing the strain energy of molecules, one can easily be seen that the strain energy of $H D N 1(n)$ and $H D N 2(n)$ is high as the values of $n$ increases. 


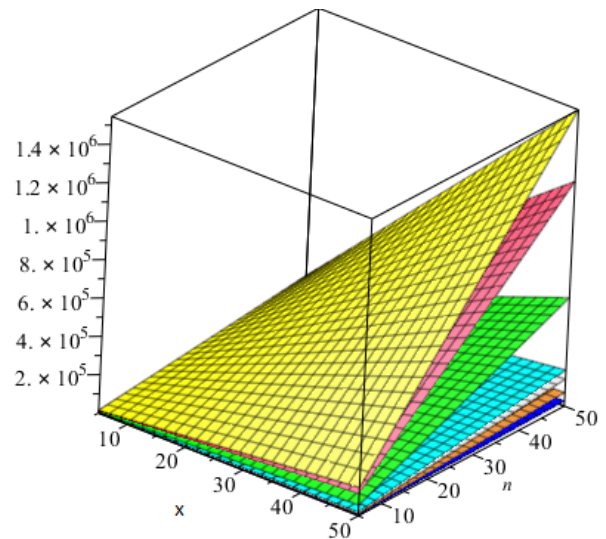

Figure 8: The comparison of all topological indices for $\operatorname{HDN} 2(n)$.

In addition, ISI index and forgotten index has much better predictive power than the predictive power of the Randić index, so the ISI index and forgotten index is more useful than the Randić index for $\alpha \in\{-1,-1 / 2\}$ as compared to the Randić index for $\alpha \in\{1,1 / 2\}$ in the case of $H D N 1(n)$ and $H D N 2(n)$.

Since the first and second Zagreb indexes were found to occur for computation of the total $\pi$-electron energy of the molecules, in the case of $H D N 1(n)$ and $H D N 2(n)$, their values provide total $\pi$-electron energy in increasing order for higher values of $n$.

For future work we propose investigation of some new type of chemical graphs and networks to compute certain degree based topological indices using polynomials.

Funding This research is supported by Quality Engineering Projects of Education Department of Anhui province (Grant No.2018jyxm1074) and Natural Science Fund of Education Department of Anhui province (Grant No.KJ2018A0598).

\section{References}

[1] Brückler F. M., Došlić T., Graovac A., Gutman I., On a class of distance-based molecular structure descriptors, Chem. Phys. Lett, 2011, 503, 336-338.

[2] Pólya G., Kombinatorische Anzahlbestimmungen für Gruppen, Graphen und chemische Verbindungen, Acta Math, 1936, 68, 145-253.

[3] Gutman I., Khadikar P. V., Rajput P. V., Karmarkar S., The Szeged index of polyacenes, J. Serb. Chem.Soc., 1995, 6759-764.

[4] Farahani M. R., Computing the hyper-Zagreb index of hexagonal nanotubes, Department of Applied Mathematics (IUST), Narmak, Tehran, Iran, 2015, 2(1), 16-18.
[5] Nikolic S., Kovacevic G., Milicevic A., Trinajstic N., The Zagreb indices 30 years after, Croat. Chem.Acta., 2003, 76, 113-124.

[6] Hosoya H., Topological Index, A Newly Proposed Quantity Characterizing the Topological Nature of Structural Isomers of Saturated Hydrocarbons, Bull. Chem. Soc. Japan, 1971, 44, 23312339.

[7] Imran M., Siddiqui M. K., Naeem M., Iqbal M. A., On Topological Properties of Symmetric Chemical Structures, Symmetry, 2018, 10, 173.

[8] Imran M., Ali M. A., Ahmad S., Siddiqui M. K., Baig A. Q., Topological Characterization of the Symmetrical Structure of Bismuth Tri-lodide, Symmetry, 2018, 10(6), 201-221.

[9] Kang S. M., Siddiqui M. K., Rehman N. A., Naeem M., Muhammad M.H., Topological Properties of 2-Dimensional SiliconCarbons, IEEE Access, 2018, 6(1), 59362-59373.

[10] Jia-Bao Liu., Siddiqui M. K., Zahid M. A., Naeem M., Baig A. Q., Topological Properties of Crystallographic Structure of Molecules, Symmetry, 2018, 10, 1-20.

[11] Gao W., Siddiqui M. K., Naeem M., Imran M., Computing multiple $A B C$ index and multiple $G A$ index of some grid graphs, Open Physic, 2018, 16(1), 588-598.

[12] Manuel P., Bharati R., Rajasingh I., Monica C. M., On minimum metric dimension of honeycomb networks, Journal of Discrete Algorithms, 2008, 6(1), 20-27.

[13] Xu D., Fan J., On the metric dimension of $H D N$, Journal of Discrete Algorithms, 2014, 26, 1-6.

[14] Imran M., Baig A. Q., Ali H., On molecular topological properties of hex-derived networks, J. Chemometrics, 2016, 30, 121-129.

[15] Gao W., Siddiqui M.K., Naeem M., Rehman N.A. Topological Characterization of Carbon Graphite and Crystal Cubic Carbon Structures, Molecules, 2017, 22, 1496-1507.

[16] Siddiqui M.K., Imran M., Ahmad A., On Zagreb indices, Zagreb polynomials of some nanostar dendrimers, Appl. Math. Comput, 2016, 280, 132-139.

[17] Siddiqui M.K., Naeem M., Rahman N.A., Imran M., Computing topological indicesof certain networks. J. Optoelectron. Adv. Mater., 2016, 18, 884-892.

[18] Siddiqui M.K., Imran M., Iqbal M.A., Molecular Descriptors of Discrete Dynamical System in Fractal and Cayley Tree Type Dendrimers, Journal of Applied Mathematics and Computing, 2019, 59(1-2), 1-16.

[19] Gao W., Farahani M.R., Jia-Bao Liu., Siddiqui M.K., harmonic degree-based index of special chemical molecular and nanotubes, U.P.B. Sci. Bull., Series B, 2019, 81(1), 1-12.

[20] Deutsch E., Klavžar S., M-Polynomial, and degree-based topological indices, Iran. J. Math. Chem, 2015, 6, 93-102.

[21] Wiener H., Structural determination of paraffin boiling points. Journal of the American Chemical Society, 1947, 1(69), 17-20.

[22] Deza M., Fowler P. W., Rassat A., Rogers K. M., Fullerenes as tiling of surfaces, J. Chem. Inf. Comput. Sci, 2000, 40, 550-558.

[23] Randić M., Characterization of molecular branching, Journal of the American Chemical Society, 1975, 97(23), 6609-6615.

[24] Böllöbás B., Erdös P., Graphs of extremal weights, Ars Combin, 1998, 50, 225-233.

[25] Gutman I, Trinastic N., Graph theory and molecular orbitals, Total $\pi$-electron energy of alternant hydrocarbons, Chem. Phys. Lett, 1972, 17, 535-538.

[26] Azari M., Iranmanesh A., Generalized Zagreb index of graphs, Studia Univ. Babes Bolyai, 2011, 56, 59-70. 
[27] Furtula B., Graovac A., Vukičević D., Augmented Zagreb index, J. Math. Chem, 2010, 48, 370--380.

[28] Farrell E. J., An introduction to matching polynomials, J. Combin. Theory Ser. B, 1979, 27, 75-86.

[29] Chou C.P., Witek H. A., Closed-form formulas for the ZhangZhang polynomials of benzenoid structures: chevrons and generalized chevrons, MATCH Commun. Math. Comput. Chem, 2014, 72, 105-124.

[30] Hassani F., Iranmanesh A., Mirzaie S., Schultz and modified Schultz polynomials of $\mathrm{C} 100$ fullerene, MATCH Commun. Math. Comput. Chem, 2013, 69, 87-92.

[31] Došlić T., Planar polycyclic graphs and their Tutte polynomials, J. Math. Chem, 2013, 51, 1599-1607.
[32] Hosoya H., On some counting polynomials in chemistry, Discrete Appl. Math, 1988, 19, 239-257.

[33] Rücker G., Rücker C., On topological indices, boiling points, and cycloalkanes, J. Chem. Inf. Comput. Sci, 1999, 39, 788-802.

[34] Munir M., Nazeer W., Rafique S., Nizami A.R., Kang S.M., Some Computational Aspects of Triangular Boron Nanotubes, Symmetry, 2016, 9, 1-6.

[35] Munir M., Nazeer W., Kang S. M., Qureshi M. I., Nizami A. R., Kwun Y. C. Some Invariants of Jahangir Graphs, Symmetry, 2017, 9, 1-15.

[36] Chen M. S., Shin K. G., Kandlur D. D., Addressing, routing, and broadcasting in hexagonal mesh multiprocessors, IEEE Trans. Comput, 1990, 39, 10-18. 